\title{
Vanishing conductivity of quantum solitons in polyacetylene
}

\author{
Leonardo Mondaini* \\ Centro Federal de Educação Tecnológica Celso Suckow da Fonseca, \\ UnED Nova Friburgo, Nova Friburgo RJ 28600-000, Brazil \\ E. C. Marindt \\ Instituto de Física, Universidade Federal do Rio de Janeiro, \\ Cx. Postal 68528, Rio de Janeiro RJ 21941-972, Brazil \\ A. A. Schmidt $\ddagger$ \\ Departamento de Matemática, Universidade Federal \\ de Santa Maria, Santa Maria RS 97105-900, Brazil
}

\begin{abstract}
Quantum solitons or polarons are supposed to play a crucial role in the electric conductivity of polyacetylene, in the intermediate doping regime. We present an exact fully quantized calculation of the quantum soliton conductivity in polyacetylene and show that it vanishes exactly. This is obtained by applying a general method of soliton quantization, based on order-disorder duality, to a $Z(2)$-symmetric complex extension of the TLM dimerization effective field theory. We show that, in this theory, polyacetylene solitons are sine-Gordon solitons in the phase of the complex field.
\end{abstract}

PACS numbers: 11.10.Kk, 11.10.Lm, 61.82.Pv

\footnotetext{
*Electronic address: leo.mondaini@pq.cnpq.br

†Electronic address: marino@if.ufrj.br

${ }^{\ddagger}$ Electronic address: alex@lana.ccne.ufsm.br
} 


\section{INTRODUCTION}

The discovery of a tremendous increase in the electrical conductivity of transpolyacetylene, when doped either with halogens or alkalis, [1] was a breakthrough of far reaching consequences in physics and chemistry. The fact that the trans-isomer occurs in two degenerate species opens the possibility of occurrence of soliton defects interconnecting them. This fact unfolded an enormous range of possibilities interconnecting many areas, including mathematics, theoretical and experimental physics. The subject has remained on

the focus of interest until recently [2]. It actually happens that such topological excitations are produced in the process of doping [3, 4]. Pure polyacetylene has one active $\pi$-electron per site and is a Peierls insulator, due to the electron-lattice interaction. It has been found that in the presence of a soliton, treated at the classical level, electron states are created in the middle of the gap, hence it is energetically favorable for the extra doped electrons to create solitons and occupy the midgap states rather than going into the conduction band.

From the very beginning the existence of three different doping regimes became clear. Firstly, for low doping concentrations (small compared to 1\%) the above picture of classical solitons works very well. The solitons are pinned by the dopant atoms, which create them with the corresponding midgap states. The conductivity is thermally activated, corresponding to a transition from the midgap to the conduction band states and can be understood quite similarly to the conductivity in semiconductors. Secondly, for high concentration of dopants (5\% to $10 \%)$ the conduction regime is clearly metallic, with an unfilled conduction band, and can be thereby understood. Thirdly, there is an intermediate regime of dopant concentration, of the order of $1 \%$, in which none of the previous models work. In this regime, the solitons become dynamic carriers of charge and a full quantum treatment of these excitations becomes unavoidable since the soliton mass is of the same order of the electron mass $[5]$.

The purpose of this work is to apply a general method of quantization of soliton excitations [6], in order to describe the conductivity of polyacetylene in the intermediate regime. In order to do that, however, the following obstacle must be removed. From the mathematical point of view, the soliton is a topologically nontrivial configuration of the dimerization - or phonon - field, which describes the lattice degrees of freedom. This, of course, is a real field, whose effective potential has $Z(2)$-symmetry and two degenerate minima that correspond to 
the two species of trans-polyacetylene. The above method of soliton quantization, however, only applies to complex fields, in the case of a multiplicative symmetry such as $Z(2)[6]$.

Therefore, in order to describe the quantum solitons of the system and specifically their role in the electric conduction in the intermediate doping regime, we propose a $Z(2)$ symmetric complex extension of the effective potential for the dimerization field. This has the same topological properties of the former and, consequently should not alter substantially the soliton physics.

In Section 2, we describe the method of soliton quantization in a theory of a complex scalar field with $Z(N)$ symmetry and show that the soliton excitations of it are sine-Gordon (SG) solitons in the phase of the complex scalar field. In Section 3, we propose the $N=2$ version of this theory as the complex extension of the effective theory for the dimerization field in polyacetylene. In Section 4, we derive an exact series expression for the quantum soliton current-current correlation function and, out of it, obtain the soliton conductivity. We show that this exactly vanishes. In conclusion, we have an exact demonstration at a full quantum level, that dynamic solitons are actually not the carriers of charge in polyacetylene. Rather, polarons, which are basically soliton-antisoliton bound states should be responsible for the transport of charge in the intermediate doping regime of polyacetylene.

\section{QUANTUM PHASE SOLITONS IN THEORIES WITH A Z(N) SYMMETRY}

We start by considering the following theory describing a complex scalar field in $(1+1)$ dimensions,

$$
\mathcal{L}=\partial_{\mu} \phi^{*} \partial^{\mu} \phi+\gamma\left(\phi^{* N}+\phi^{N}\right)-\eta\left(\phi^{*} \phi\right)^{M}
$$

where $N$ and $M$ are integers and $\gamma$ and $\eta$ are real parameters. This is invariant under the $Z(N)$ transformation: $\phi(x, t) \rightarrow e^{i \frac{2 \pi}{N}} \phi(x, t)$. The choice $\gamma>0$, implies the spontaneous breakdown of the $Z(N)$ symmetry. In this case, the theory will have degenerate vacua and soliton excitations. A full quantum theory of these solitons was developed in [6, 7, 8]. This includes an explicit expression for the soliton creation operator, namely

$$
\mu(x)=\exp \left\{-\frac{2 \pi}{N} \int_{x, C}^{\infty} d \xi_{\nu} \epsilon^{\mu \nu} \phi^{*}(\xi) \stackrel{\leftrightarrow}{\partial}_{\mu} \phi(\xi)\right\}
$$

and a general expression for its local Euclidean correlation functions [6, 7, 8],

$$
\left\langle\mu(x) \mu^{\dagger}(y)\right\rangle=\mathcal{N} \int \mathcal{D} \phi^{*} \mathcal{D} \phi \exp \left\{-\int d^{2} z\left[\left(D_{\mu} \phi\right)^{*}\left(D_{\mu} \phi\right)+V\left(\phi^{*}, \phi\right)\right]\right\},
$$


where

$$
D_{\mu}=\partial_{\mu}-i \alpha A_{\mu}, \quad A_{\mu}(z, C)=\int_{x, C}^{y} d \xi_{\nu} \epsilon^{\mu \nu} \delta^{2}(z-\xi) .
$$

In the above expression, $V$ is the potential of an arbitrary Lagrangian and the integral is taken along an arbitrary curve $C$, connecting $x$ and $y$. It can be shown, however, that (3) is independent of the chosen curve.

We are going to show in what follows that these quantum solitons may be identified with SG quantum solitons in the phase of the field $\phi$.

Using the polar representation for $\phi$, namely, $\phi(x, t)=\rho(x, t) e^{i \theta(x, t)}$, where $\rho$ and $\theta$ are real fields, we can rewrite the above Lagrangian as

$$
\mathcal{L}=\partial_{\mu} \rho \partial^{\mu} \rho+\rho^{2} \partial_{\mu} \theta \partial^{\mu} \theta+2 \gamma \rho^{N} \cos N \theta-\eta \rho^{2 M}
$$

In what follows, we will be interested in the topological properties of the theory. As we shall argue, these are not affected by $\rho$ fluctuations, hence, from now on we will make the constant $\rho$ approximation,

$$
\rho(x, t)=\rho_{0}, \quad \rho_{0} \text { constant }
$$

Using this in (5) we get

$$
\mathcal{L}=\rho_{0}^{2} \partial_{\mu} \theta \partial^{\mu} \theta+2 \gamma \rho_{0}^{N} \cos N \theta-\eta \rho_{0}^{2 M}
$$

which is a SG Lagrangian in $\theta$.

We conclude that, in the constant- $\rho$ approximation, the theories given by (11) will present SG solitons in the phase of the complex scalar field $\phi$. The corresponding topological current will be

$$
J^{\mu}=\epsilon^{\mu \nu} \partial_{\nu} \theta
$$

which is associated to the topological charge operator

$$
\mathcal{Q}=\int_{-\infty}^{\infty} d x^{\prime} J^{0}=\int_{-\infty}^{\infty} d x^{\prime} \partial_{x^{\prime}} \theta\left(x^{\prime}, t\right)=\theta(+\infty, t)-\theta(-\infty, t)
$$

We see that topological properties are related to large $\theta$ fluctuations and, therefore, the constant $\rho$ approximation should not interfere in such properties.

In order to explicitly confirm the fact that the soliton operators introduced in [6, 7, 8] are indeed creation operators of quantum solitons in the phase of the field $\phi$, let us explicitly 
evaluate the commutation relation between the quantum soliton creation operator and the topological charge.

Observe that this is nothing but the Mandelstam creation operator of quantum solitons in the SG model [9], as it should. Then, using canonical commutation relations we readily find

$$
\begin{array}{r}
{[\mathcal{Q}, \mu]=\left\{-i \frac{2 \pi}{N} \int_{-\infty}^{\infty} d x^{\prime} \partial_{x^{\prime}} \int_{x}^{\infty} d \xi_{1}\left[\theta\left(x^{\prime}, t\right), \quad \pi_{\theta}\left(\xi_{1}, t\right)\right]\right\} \mu} \\
=\frac{2 \pi}{N} \mu .
\end{array}
$$

Eq. (10) implies that the operator $\mu$ creates eigenstates of the topological charge $\mathcal{Q}$, with eigenvalue $2 \pi / N$, thus proving that the quantum solitons occurring in the theory described by (11) are indeed phase solitons. Correlation functions of these quantum soliton excitations

have been calculated elsewhere [10, 11, 12]. In Section 4, we will show that the relevant quantum correlators for the calculation of the conductivity will be the soliton current-current correlators.

\section{THE CASE N=2: A MODEL FOR POLYACETYLENE}

In this section, we are going to propose a phenomenological theory for polyacetylene that will enable us to compute quantum soliton correlation functions and in particular the correlation functions of quantum solitonic current operators. As we shall see the standard field theory model for this polymer unfortunately does not allow the application of the method of soliton quantization described in the previous section. For this reason, we will propose a phenomenological alternative.

Polyacetylene is described by the Su-Schrieffer-Heeger (SSH) model [3, 4], whose field theory version is the Takayama-Lin-Liu-Maki (TLM) model [13], described by the Hamiltonian

$$
\begin{aligned}
H_{T L M}= & \int d x \Psi_{s}^{\dagger}(x)\left[-i \hbar v_{F} \sigma_{3} \partial_{x}+\Delta(x) \sigma_{1}\right] \Psi_{s}(x) \\
& +\left(2 \pi \hbar v_{F} \lambda\right)^{-1} \int d x\left[\dot{\Delta}^{2}(x) / \Omega_{0}^{2}+\Delta^{2}(x)\right] .
\end{aligned}
$$

In the above expression, $\Psi_{s}(x)$ is a two-component Dirac fermion field, associated to the $\pi$-electrons and $\Delta(x)$ is a real scalar field - the dimerization field - associated to the lattice 
degrees of freedom, namely, the phonons. Furthermore, $\sigma_{i}$ are the Pauli matrices, $v_{F}$ is the Fermi velocity, $\lambda$ is the dimensionless electron-phonon coupling constant, and $\Omega_{0}$ is the bare optical-phonon frequency.

Integrating over the fermion field in the previous expression, we obtain an effective theory for the phonon field $\Delta(x)$, whose potential is a $\mathrm{Z}(2)$ symmetric double-well [14, 15, 16]. The two degenerate minima of this potential correspond to the two degenerate dimerizations of trans-polyacetylene. The equivalent calculation has also been performed within the SSH model [3, 4], also leading to a degenerate double-well effective potential for the dimerization variable.

The double-well potential for the effective dimerization field implies the existence of soliton excitations in the theory. It has been shown that these, indeed, are introduced by doping polyacetylene with halogen or alkali atoms [1].

As we have argued in the Introduction, in the regime of intermediate doping, a full quantum treatment of the soliton excitations is unavoidable. Hence, one would be naturally inclined to use the method of soliton quantization for theories with a $Z(N)$ symmetry, described in the previous section, for the case $N=2$. Nevertheless, one immediately realizes that the method is not applicable for a real field such as the dimerization field $\Delta(x)$. Indeed, for a real field the exponent of the soliton operator (2) vanishes, making $\mu$ trivial and the soliton correlator (3) no longer makes sense, since we cannot couple the external field $A_{\mu}$ to a real field. Furthermore, for a real field, $\theta=0$, hence the topological current and the respective topological charge cannot be defined as in (8) and (9). The above method of soliton quantization, in the case of a multiplicative symmetry [6], only applies to complex fields.

In order to conciliate this fact with the knowledge that the effective theory for the real $\Delta(x)$-field is a degenerate double-well with a $Z(2)$ symmetry, we propose a complex extension $\phi(x)$ of the $\Delta(x)$-field, governed by the Lagrangian (1) with $N=M=2$. The corresponding potential is

$$
V\left(\phi^{*}, \phi\right)=-\gamma\left(\phi^{* 2}+\phi^{2}\right)+\eta\left(\phi^{*} \phi\right)^{2}
$$

or, in terms of polar fields,

$$
V(\rho, \theta)=-2 \gamma \rho^{2} \cos 2 \theta+\eta \rho^{4}
$$


As we can see, there are two degenerate minima at

$$
\left(\rho_{0}, \theta_{0}\right)=\left\{\begin{array}{l}
\left(\sqrt{\frac{\gamma}{\eta}}, 0\right) \\
\left(\sqrt{\frac{\gamma}{\eta}}, \pi\right)
\end{array}\right.
$$

where the potential has the value $V\left(\rho_{0}, \theta_{0}\right)=-\gamma^{2} / \eta$. Applying, then, the constant $\rho$ approximation

$$
\rho(x, t) \simeq \rho_{0}=\sqrt{\frac{\gamma}{\eta}}
$$

and adding $\gamma^{2} / \eta$ we get the following SG potential for the phase field $\theta$,

$$
V(\theta)=\frac{2 \gamma^{2}}{\eta}(1-\cos 2 \theta)
$$

The associated classical solitonic excitations will be

$$
\theta(x)= \pm 2 \arctan \exp \left[\sqrt{\gamma}\left(x-x_{0}\right)\right]
$$

where the plus and minus signs correspond respectively to a soliton $\left(\theta_{s}\left(x-x_{0}\right)\right)$ and an anti-soliton in the phase of the $\phi$-field. We see that

$$
\lim _{x \rightarrow-\infty} \theta_{s}\left(x-x_{0}\right)=0 \quad \text { and } \quad \lim _{x \rightarrow \infty} \theta_{s}\left(x-x_{0}\right)=\pi
$$

namely, the phase soliton connects the minima of the potential (13), when $\rho=\sqrt{\gamma / \eta}$.

The actual potential for the dimerization field $\Delta(x)$ and the $Z(2)$-symmetric complex potential for the field $\phi$, given by (13), both possess the same topology, related to the $Z(2)$-symmetry. It is therefore reasonable to expect the same topological properties in both theories, especially those concerning solitons. We may adjust the parameters in such a way that the minima of the real field potential coincide with those of the complex one.

What we are doing is quite similar to what is done when we use complex functions in order to describe the EM field. The physical E and B fields will correspond to the real part thereof. The dimerization field $\Delta$ of the TLM model is the real part of our $\phi$. As we know, for the polyacetilene soliton, we have the $\Delta$-field varying from $-\Delta_{0}$ to $\Delta_{0}$ in between the two minima.

A SG soliton in theta would have the phase of the $\phi$ field varying from $\pi$ to 0 , implying $\phi$ would vary between $-\rho_{0}$ and $\rho_{0}$. Thus, identifying $\rho_{0}$ with $\Delta_{0}$, we can figure out the relation between the sine-Gordon solitons of our model and the polyacetylene solitons: the 
real part of the complex field $\phi$ for the configuration having a SG soliton in its phase will be in the same topological class as the $\phi^{4}$-like soliton of polyacetylene. Since for each SG soliton there is a soliton in polyacetylene we may identify the SG-soliton current with the polymer soliton current. We are going to use, therefore, this complex extension in order to study the quantum properties of the soliton excitations by means of the method of soliton quantization described in the previous section. We will use, in particular, the SG soliton current for calculating the conductivity.

\section{THE QUANTUM SOLITON CURRENT CORRELATOR AND CONDUCTIV- ITY}

In this section, we are going to obtain an exact series expression for the soliton dcconductiviy in our model for polyacetylene. For this purpose, the starting point is the well-known Kubo formula [17],

$$
\sigma_{s}^{i j}=\lim _{\omega \rightarrow 0} \lim _{k \rightarrow 0} \frac{1}{\omega} \operatorname{Im}\left[\left\langle J^{i} J^{j}\right\rangle_{r e t}(\omega, k)\right],
$$

where $\left\langle J^{i} J^{j}\right\rangle_{r e t}(\omega, k)$ is the retarded, Minkowski space, correlation function of the spatial component of the soliton current operator $J^{\mu}$ given by (8).

We want to evaluate the above current-current correlator within our field theory model for polyacetylene. The strategy will be to derive a generating functional for such current correlators in our field theory model. For this purpose, we introduce the identity

$$
\begin{array}{r}
1=\int \mathcal{D} J^{\mu} \delta\left[J^{\mu}-\epsilon^{\mu \nu} \partial_{\nu} \theta\right] \\
=\int \mathcal{D} J^{\mu} \mathcal{D} \lambda_{\mu} \exp \left\{i \int d^{2} z\left(J^{\mu}-\epsilon^{\mu \nu} \partial_{\nu} \theta\right) \lambda_{\mu}\right\},
\end{array}
$$

in the Euclidian vacuum functional associated to the Lagrangian (17), obtaining

$$
\begin{aligned}
& \mathcal{Z}=\mathcal{Z}_{0}^{-1} \int \mathcal{D} J^{\mu} \mathcal{D} \lambda_{\mu} \mathcal{D} \theta \exp \left\{-\frac{1}{\hbar v_{S}} \int d^{2} z\left[\rho_{0}^{2} \partial_{\mu} \theta \partial_{\mu} \theta\right.\right. \\
&\left.\left.-2 \gamma \rho_{0}^{N} \cos N \theta-i \hbar v_{S}\left(J^{\mu}-\epsilon^{\mu \nu} \partial_{\nu} \theta\right) \lambda_{\mu}\right]\right\}
\end{aligned}
$$

where we used expression (8) for the soliton current. Notice that in the expression above, we no longer make $\hbar=c=1$. Actually, since the $\phi$-field theory replaces the effective theory for the phonon field $\Delta$ in the TLM model, we substitute $c$ for $v_{S}$, the speed of sound in the polymer. 
We now integrate over $\theta$ and $\lambda_{\mu}$, thereby obtaining the partition function expressed as the functional integral of the exponential of an effective $J^{\mu}$ action [18]. The $\theta$ integral may be done by the usual expansion in powers of the cosine term [19], or equivalently, in powers of $\rho_{0}^{N}$. The resulting functional integrals, both in $\theta$ and $\lambda_{\mu}$ are quadratic and the final result is

$$
\begin{array}{r}
\mathcal{Z}=\mathcal{N} \sum_{m=0}^{\infty} \frac{\left(\frac{\gamma \rho_{0}^{N}}{\hbar w_{S}}\right)^{2 m}}{(m !)^{2}} \int \prod_{i=1}^{2 m} d^{2} z_{i} \int \mathcal{D} J^{\mu} \exp \left\{-\frac{1}{2} \int d^{2} z d^{2} z^{\prime}\right. \\
\times J^{\mu}(\vec{z})\left[\frac{2 \rho_{0}^{2}}{\hbar v_{S}} \delta^{\mu \nu} \delta^{2}\left(\vec{z}-\overrightarrow{z^{\prime}}\right)\right] J^{\nu}\left(\overrightarrow{z^{\prime}}\right) \\
\left.+\int d^{2} z\left[i N \sum_{i=1}^{2 m} \lambda_{i} \epsilon^{\mu \alpha} \partial_{\alpha} G\left(\overrightarrow{z_{i}}-\vec{z}\right)\right] J^{\mu}(\vec{z})\right\},
\end{array}
$$

where $\lambda_{i}=1$ for $1 \leq i \leq m$ and $\lambda_{i}=-1$ for $n+1 \leq i \leq 2 m$ and $G(\vec{z})$ is the Euclidian Green function of the two-dimensional (2D) free massless scalar theory, which appears naturally since the expansion in powers of the cosine term is an expansion around such theory.

We note at this point that, should we integrate the above expression over $J^{\mu}$, we would obtain the usual Coulomb gas representation for the vacuum functional of the SG theory [20]. Conversely, the expression for the generating functional of current correlators can be obtained by the usual procedure of adding a linear coupling with a source $K_{\mu}$ in the exponent of the integrand in the previous espression, namely,

$$
\begin{array}{r}
\mathcal{Z}\left[K_{\mu}\right]=\mathcal{N} \sum_{m=0}^{\infty} \frac{\left(\frac{\gamma \rho_{0}^{N}}{\hbar v_{S}}\right)^{2 m}}{(m !)^{2}} \int \prod_{i=1}^{2 m} d^{2} z_{i} \int \mathcal{D} J^{\mu} \exp \left\{-\frac{1}{2} \int d^{2} z d^{2} z^{\prime}\right. \\
\times J^{\mu}(\vec{z})\left[\frac{2 \rho_{0}^{2}}{\hbar v_{S}} \delta^{\mu \nu} \delta^{2}\left(\vec{z}-\overrightarrow{z^{\prime}}\right)\right] J^{\nu}\left(\overrightarrow{z^{\prime}}\right) \\
\left.+\int d^{2} z\left[i N \sum_{i=1}^{2 m} \lambda_{i} \epsilon^{\mu \alpha} \partial_{\alpha} G\left(\overrightarrow{z_{i}}-\vec{z}\right)+\frac{K_{\mu}}{\hbar v_{S}}\right] J^{\mu}(\vec{z})\right\}
\end{array}
$$

$\mathcal{Z}\left[K_{\mu}\right]$ is the desired generating functional of $J^{\mu}$ correlators. Indeed, we have

$$
\left\langle J^{\mu}(\vec{x}) J^{\nu}(\vec{y})\right\rangle=\left.\frac{\left(\hbar v_{S}\right)^{2}}{\mathcal{Z}} \frac{\delta^{2} \mathcal{Z}\left[K_{\mu}\right]}{\delta K_{\mu}(\vec{x}) \delta K_{\nu}(\vec{y})}\right|_{K_{\mu}=0} .
$$


Now, integrating (23) in $J^{\mu}$, we get

$$
\begin{array}{r}
\mathcal{Z}\left[K_{\mu}\right]=\sum_{m=0}^{\infty} \frac{\left(\frac{\gamma \rho_{0}^{N}}{\hbar v_{S}}\right)^{2 m}}{(m !)^{2}} \int \prod_{i=1}^{2 m} d^{2} z_{i} \\
\times \exp \left\{\frac{1}{2} \int d^{2} z d^{2} z^{\prime}\left[i N \sum_{i=1}^{2 m} \lambda_{i} \epsilon^{\mu \alpha} \partial_{\alpha} G\left(\vec{z}_{i}-\vec{z}\right)+\frac{K_{\mu}}{\hbar v_{S}}\right]\right. \\
\left.\times\left[\frac{\hbar v_{S}}{2 \rho_{0}^{2}} \delta^{\mu \nu} \delta^{2}\left(\vec{z}-\overrightarrow{z^{\prime}}\right)\right]\left[i N \sum_{j=1}^{2 m} \lambda_{j} \epsilon^{\nu \beta} \partial_{\beta}{ }^{\prime} G\left(\vec{z}_{j}-\overrightarrow{z^{\prime}}\right)+\frac{K_{\nu}{ }^{\prime}}{\hbar v_{S}}\right]\right\} \\
\times \exp \left\{-\frac{N^{2} \hbar v_{S}}{4 \rho_{0}^{2}} \sum_{i=1}^{2 m} \frac{\left(\frac{\gamma \rho_{0}^{N}}{\hbar v_{S}}\right)^{2 m}}{(m !)^{2}} \int \prod_{j=1}^{2 m} \lambda_{j}^{2 m} d^{2} z_{i}\right. \\
\left.\times \exp \left\{\frac{1}{4 \rho_{0}^{2} \hbar v_{S}} \int \vec{z}_{j}\right)\right\} \\
+\frac{i N}{2 \rho_{0}^{2}} \sum_{i=1}^{2 m} d^{2} d^{2} z^{\prime} K_{\mu}(\vec{z}) \delta^{2}\left(\vec{z}-\overrightarrow{z^{\prime}}\right) K_{\mu}\left(\overrightarrow{z^{\prime}}\right)
\end{array}
$$

Evaluating the functional derivatives in (24), we obtain

$$
\begin{array}{r}
\left\langle J^{\mu}(\vec{x}) J^{\nu}(\vec{y})\right\rangle=\mathcal{Z}^{-1} \sum_{m=0}^{\infty} \frac{\left(\frac{\gamma \rho_{0}^{N}}{\hbar v_{S}}\right)^{2 m}}{(m !)^{2}} \int \prod_{i=1}^{2 m} d^{2} z_{i} \\
\times \exp \left\{-\frac{N^{2} \hbar v_{S}}{4 \rho_{0}^{2}} \sum_{i=1}^{2 m} \lambda_{i} \sum_{j=1}^{2 m} \lambda_{j} G\left(\vec{z}_{i}-\vec{z}_{j}\right)\right\} \\
\times\left\{\frac{\hbar v_{S}}{2 \rho_{0}^{2}} \delta^{\mu \nu} \delta^{2}(\vec{x}-\vec{y})-\frac{N^{2}\left(\hbar v_{S}\right)^{2}}{4 \rho_{0}^{4}} \sum_{j=1}^{2 m} \lambda_{j} G\left(\vec{z}_{j}\right)\right. \\
\left.\times \sum_{i=1}^{2 m} \lambda_{i}\left(\delta^{\mu \nu} \partial_{\alpha}^{(y)} \partial_{\alpha}^{(x)}-\partial_{\mu}^{(y)} \partial_{\nu}^{(x)}\right) G\left(\vec{z}_{i}+(\vec{x}-\vec{y})\right)\right\},
\end{array}
$$

where we have made the shift of variable $\vec{z}_{i(j)} \rightarrow \vec{z}_{i(j)}-\vec{x}$. 
We now perform the Fourier transform in the variable $\vec{\chi} \equiv \vec{x}-\vec{y}$, arriving at

$$
\begin{array}{r}
\left\langle J^{\mu} J^{\nu}\right\rangle(\vec{k})=\mathcal{Z}^{-1} \sum_{m=0}^{\infty} \frac{\left(\frac{\gamma \rho_{0}^{N}}{\hbar v_{S}}\right)^{2 m}}{(m !)^{2}} \int \prod_{i=1}^{2 m} d^{2} z_{i} \\
\times \exp \left\{-\frac{N^{2} \hbar v_{S}}{4 \rho_{0}^{2}} \sum_{i=1}^{2 m} \lambda_{i} \sum_{j=1}^{2 m} \lambda_{j} G\left(\vec{z}_{i}-\vec{z}_{j}\right)\right\} \\
\times\left\{\frac{\hbar v_{S}}{2 \rho_{0}^{2}} \delta^{\mu \nu}-\frac{N^{2}\left(\hbar v_{S}\right)^{2}}{4 \rho_{0}^{4}} \sum_{j=1}^{2 m} \lambda_{j} G\left(\vec{z}_{j}\right)\right. \\
\left.\times\left\{\sum_{i=1}^{2 m} \lambda_{i} e^{-i \vec{k} \cdot \vec{z}_{i}}\left(\frac{\delta^{\mu \nu} \vec{k}^{2}-k^{\mu} k^{\nu}}{\vec{k}^{2}}\right)\right\}\right\},
\end{array}
$$

where $\vec{k} \equiv(k, \omega)$ and we used the fact that

$$
\int d^{2} \chi\left(\delta^{\mu \nu} \partial_{\alpha}^{(y)} \partial_{\alpha}^{(x)}-\partial_{\mu}^{(y)} \partial_{\nu}^{(x)}\right) G\left(\vec{\chi}+\vec{z}_{i}\right) e^{i \vec{k} \cdot \vec{\chi}}=e^{-i \vec{k} \cdot \vec{z}_{i}}\left(\frac{\delta^{\mu \nu} \vec{k}^{2}-k^{\mu} k^{\nu}}{\vec{k}^{2}}\right) .
$$

From (27) we can get $\left\langle J^{i} J^{j}\right\rangle_{\text {ret }}(\omega, k)$, by following the prescription given in [17], for the retarded Green function, which includes the change of variables (recalling that $\vec{z} \equiv(z, \tau)$ )

$$
i \tau \rightarrow-v_{S} t, \quad i \omega \rightarrow \frac{\omega}{v_{S}}+i \delta
$$

and the limit $\delta \rightarrow 0$. The soliton conductivity, then, is given by

$$
\sigma_{s}=\lim _{\delta \rightarrow 0} \lim _{\omega \rightarrow 0} \lim _{k \rightarrow 0} \frac{1}{\omega} \operatorname{Im}\left[\langle J J\rangle_{r e t}(\omega, k)\right]
$$

Taking the above limits, we obtain, after some algebra

$$
\begin{aligned}
& \sigma_{s}=\mathcal{Z}^{-1} \frac{N^{2}\left(\hbar v_{S}\right)^{2}}{4 \rho_{0}^{4}} \sum_{m=1}^{\infty} \frac{\left(\frac{\gamma \rho_{0}^{N}}{\hbar v_{S}}\right)^{2 m}}{(m !)^{2}} \int \prod_{i=1}^{2 m}\left(i v_{S} d t_{i}\right)\left(d z_{i}\right) \\
& \times \exp \left\{-\frac{N^{2} \hbar v_{S}}{4 \rho_{0}^{2}} \sum_{i=1}^{2 m} \lambda_{i} \sum_{j=1}^{2 m} \lambda_{j} G\left(\vec{z}_{i}-\vec{z}_{j}\right)\right\} \\
& \times\left\{\sum_{j=1}^{2 m} \lambda_{j} G\left(\vec{z}_{j}\right)\right\}\left\{\sum_{i=1}^{2 m} \lambda_{i} t_{i}\right\} .
\end{aligned}
$$

The non-transverse part of (27) is a non-physical "zero-point" term, which must be subtracted from the current correlator. Anyway it would not contribute to the conductivity because it is real. 
The temperature dependence of the soliton conductivity may now be obtained by the usual methodology, through which we are led to the version of (31) having finite integration regions $0<\tau_{i}<\beta\left(\beta=\hbar v_{S} / k_{B} T\right)$ in the Euclidian time,

$$
\begin{array}{r}
\sigma_{s}^{E}(T)=i \sigma_{s}(T) \\
=\frac{-i \mathcal{Z}^{-1}}{v_{S}} \frac{N^{2}\left(\hbar v_{S}\right)^{2}}{4 \rho_{0}^{4}} \sum_{m=1}^{\infty} \frac{\left(\frac{\gamma \rho_{0}^{N}}{\hbar v_{S}}\right)^{2 m}}{(m !)^{2}} \int_{0}^{\frac{\hbar v_{S}}{k_{B} T}} \int_{-\infty}^{\infty} \prod_{i=1}^{2 m} d \tau_{i} d z_{i} \\
\times \exp \left\{-\frac{N^{2} \hbar v_{S}}{4 \rho_{0}^{2}} \sum_{i=1}^{2 m} \lambda_{i} \sum_{j=1}^{2 m} \lambda_{j} G_{T}\left(\vec{z}_{i}-\vec{z}_{j}\right)\right\} \\
\times\left\{\sum_{j=1}^{2 m} \lambda_{j} G_{T}\left(\vec{z}_{j}\right)\right\}\left\{\sum_{i=1}^{2 m} \lambda_{i} \tau_{i}\right\} .
\end{array}
$$

In the above expression, the free massless Green function has been replaced by the corresponding function at a finite temperature $T$, namely, $G_{T}(\vec{z})(\vec{z} \equiv(z, \tau))$. This is a natural consequence of the frequency quantization in the presence of a finite interval for $\tau$.

The thermal Euclidian Green function of the 2D free massless scalar theory in coordinate space has been evaluated in [21] and is given by

$$
G_{T}(\vec{z})=-\frac{1}{4 \pi} \ln \left\{\frac{\mu_{0}^{2} \beta^{2}}{\pi^{2}}\left[\cosh \left(\frac{2 \pi k_{B} T}{\hbar v_{S}} z\right)-\cos \left(\frac{2 \pi k_{B} T}{\hbar v_{S}} \tau\right)\right]\right\} .
$$

Furthermore, the one-dimensional electrical conductivity is related to the quantum soliton conductivity presented in (32) by

$$
\sigma_{e}=\left(\frac{e^{2} v_{S}}{\hbar}\right) \sigma_{s}
$$

In order to obtain a result that could be compared with experimental data, i.e., the threedimensional electrical conductivity, we must divide the above expression by the cross-section area of the polyacetylene fibers, namely, $A \simeq \pi \cdot 10^{4} \AA^{2}[22]$. Then, we have

$$
\sigma=\frac{\sigma_{e}}{A} .
$$

In what follows, we will explicitly demonstrate that Eq. (32) yields an exactly vanishing quantum soliton conductivity. First of all, let us change our notation by defining

$$
\vec{z}_{i}=\left\{\begin{array}{l}
\vec{z}_{i}^{+} \equiv\left(z_{i}^{+}, \tau_{i}^{+}\right), \quad \text { for } 1 \leq i \leq m ; \\
\vec{z}_{i}^{-} \equiv\left(z_{i}^{-}, \tau_{i}^{-}\right), \quad \text { for } m+1 \leq i \leq 2 m .
\end{array}\right.
$$


Using the above notation and making the change of variables $\tau_{i}^{+(-)} \rightarrow\left(\frac{2 \pi k_{B} T}{\hbar v_{S}}\right) \tau_{i}^{+(-)}$, we may then rewrite Eq. (32) as

$$
\begin{aligned}
\sigma_{s}^{E}(T)= & i \sigma_{s}(T)=\frac{-i \mathcal{Z}^{-1}}{v_{S}} \frac{N^{2}\left(\hbar v_{S}\right)^{2}}{4 \rho_{0}^{4}} \sum_{m=1}^{\infty} \frac{\left(\frac{\gamma \rho_{0}^{N}}{\hbar v_{S}}\right)^{2 m}}{(m !)^{2}}\left(\frac{\hbar v_{S}}{2 \pi k_{B} T}\right)^{2 m+1} \\
& \times\left(\prod_{i=1}^{m} \int_{0}^{2 \pi} d \tau_{i}^{+} \int_{0}^{2 \pi} d \tau_{i}^{-}\right) \Xi_{m}\left(\tau_{1}^{+}, \ldots, \tau_{m}^{+} ; \tau_{1}^{-}, \ldots, \tau_{m}^{-}\right)
\end{aligned}
$$

where

$$
\begin{array}{r}
\Xi_{m}\left(\tau_{1}^{+}, \ldots, \tau_{m}^{+} ; \tau_{1}^{-}, \ldots, \tau_{m}^{-}\right) \\
=\left\{\sum_{i=1}^{m}\left(\tau_{i}^{+}-\tau_{i}^{-}\right)\right\}\left(\prod_{i=1}^{m} \int_{-\infty}^{\infty} d z_{i}^{+} \int_{-\infty}^{\infty} d z_{i}^{-}\right) \\
\times \exp \left\{-\frac{N^{2} \hbar v_{S}}{4 \rho_{0}^{2}} \sum_{i, j=1}^{m}\left[G_{T}\left(\vec{z}_{i}^{+}-\vec{z}_{j}^{+}\right)+G_{T}\left(\vec{z}_{i}^{-}-\vec{z}_{j}^{-}\right)\right.\right. \\
\left.\left.-G_{T}\left(\vec{z}_{i}^{+}-\vec{z}_{j}^{-}\right)-G_{T}\left(\vec{z}_{i}^{-}-\vec{z}_{j}^{+}\right)\right]\right\} \\
\times\left\{\sum_{j=1}^{m}\left[G_{T}\left(\vec{z}_{j}^{+}\right)-G_{T}\left(\vec{z}_{j}^{-}\right)\right]\right\},
\end{array}
$$

in which, after re-scaling $\tau$,

$$
G_{T}(\vec{z})=-\frac{1}{4 \pi} \ln \left\{\frac{\mu_{0}^{2} \beta^{2}}{\pi^{2}}\left[\cosh \left(\frac{2 \pi k_{B} T}{\hbar v_{S}} z\right)-\cos \tau\right]\right\} .
$$

We will now show that the quantum soliton conductivity vanishes exactly. For this, we make the change of variables $\tau_{i}^{+(-)} \rightarrow 2 \pi-\tau_{i}^{+(-)}$in the $\tau$-integrals in (37). Since

$$
\begin{array}{r}
\Xi_{m}\left(2 \pi-\tau_{1}^{+}, \ldots, 2 \pi-\tau_{m}^{+} ; 2 \pi-\tau_{1}^{-}, \ldots, 2 \pi-\tau_{m}^{-}\right) \\
=-\Xi_{m}\left(\tau_{1}^{+}, \ldots, \tau_{m}^{+} ; \tau_{1}^{-}, \ldots, \tau_{m}^{-}\right),
\end{array}
$$

the announced result immediately follows.

\section{CONCLUSION}

The application of a general method of soliton quantization, based on order-disorder duality, to a $Z(2)$ symmetric complex extension of the effective field theory for the dimerization field of the TLM model for polyacetylene has yielded an exactly vanishing result for the quantum soliton conductivity. This strongly suggests that dynamic solitons are not the 
charge carriers in polyacetylene in the intermediate doping regime. The natural candidates are polarons. However, as it happens in the case of solitons, which were studied in the present work, a full quantum treatment is required in order to derive a reliable expression for the polaron conductivity as a function of the temperature. We are presently investigating the quantum polaronic conductivity in this system.

This work has been supported in part by CNPq and FAPERJ. LM and AAS were supported by CNPq. ECM was partially supported by CNPq.

[1] Chiang C K et al. 1977 Phys. Rev. Lett. 391098

[2] Hultell M and Stafstrom S 2007 Phys. Rev. B 75104304 ; Di B, An Z, Li Y C and Wu C Q 2007 Europhysics Letters 79 17002; Li X et al. 2006 Phys. Rev. B 74 172301; Okamoto H and Ono Y 2005 J. Phys. Soc. Japan, 74 1006; Tanner D B et al. 2004 Synthetic Metals 141 75; Johansson A and Stafstrom S 2002 Phys. Rev. B 65045207 Chiang C K et al. 1977 Phys. Rev. Lett. 391098

[3] Su W P, Schrieffer J R and Heeger A J 1979 Phys. Rev. Lett. 421698

[4] Su W P, Schrieffer J R and Heeger A J 1980 Phys. Rev. B 222099

[5] Heeger A J et al. 1988 Rev. Mod. Phys. 60781

[6] Marino E C 1990 Applications of Statistical and Field Theory Methods to Condensed Matter ed D Baeriswyl, A R Bishop and J Carmelo (New York: Plenum Press)

[7] Marino E C and Swieca J A 1980 Nucl. Phys. B 170[FS1] 175

[8] Marino E C, Schroer B and Swieca J A 1982 Nucl. Phys. B 200[FS4] 473

[9] Mandelstam S 1975 Phys. Rev. D 113026

[10] Mondaini L and Marino E C 2005 J. Stat. Phys. 118767

[11] Mondaini L and Marino E C 2006 J. Phys. A: Math. Gen. 39967

[12] Mondaini L and Marino E C 2008 Mod. Phys. Lett. A 23761

[13] Takayama H, Lin-Liu Y R and Maki K 1980 Phys. Rev. B 212388

[14] Campbell D K and Bishop A R 1981 Phys. Rev. B 244859

[15] Campbell D K and Bishop A R 1982 Nucl. Phys. B 200[FS4] 297

[16] Gross D J and Neveu A 1974 Phys. Rev. D 103235

[17] Mahan G D 2000 Many-Particle Physics (New York: Kluwer Academic/Plenum Publishers) 
[18] Mondaini L 2006 Ph.D. thesis (Rio de Janeiro: IF-UFRJ)

[19] Lima-Santos A and Marino E C 1989 J. Stat. Phys. 55157

[20] Samuel S 1978 Phys. Rev. D 181916

[21] Delépine D, González Felipe R and Weyers J 1998 Phys. Lett. B 419296

[22] Shirakawa H and Ikeda S 1980 Synth. Met. 1175 